\title{
Oxygen/Ozone Therapy: a promising approach for the treatment of biphosphonate-related osteonecrosis of the jaw
}

\author{
Girolamo Donati $^{1}$, Valentina Laura Donati ${ }^{2}$, Carlo Alberto Rossi \\ 1 ASST Fatebenefratelli Sacco \\ 2 Technical University of Denmark
}

Funding: The author(s) received no specific funding for this work.

Potential competing interests: The author(s) declared that no potential competing interests exist.

\section{Abstract}

Biphosphonate-related osteonecrosis of the jaw (BRONJ), a side effect following biphosphonate treatment, consists of a progressive bone destruction in the maxillofacial region and it is traditionally treated with antibiotic therapy and surgical removal of the necrotic bone. Among the studied alternative therapies, oxygen/ozone therapy has shown positive results.

In the present study, we evaluated how the ozone/oxygen therapy $\left(\mathrm{O}_{2} / \mathrm{O}_{3}\right.$ mixture from medical oxygen) and the debridement with piezoelectric surgery may improve the treatment of BRONJ reaching a complete healing of the lesion. The experimental protocol consisted of the application of an $\mathrm{O}_{2} / \mathrm{O}_{3}$ mixture twice a week for 10 weeks before the debridement surgery and afterwards. Clinical and radiological parameters were used to evaluate the lesions. Following up on the preliminary data recently published by Goker et al. (2020) ${ }^{[1]}$, here we present the updated case reports. Until now, sixteen patients with BRONJ at stage 1 and 2 (classified according to the SICMF-SIPMO - Sistema di stadiazione clinica e radiologica) have been included in the treatment. After the $\mathrm{O}_{2} / \mathrm{O}_{3}$ therapy, 12 patients recovered from osteonecrosis and, in seven among these, the spontaneous expulsion of the bone fragment was observed. Relapses were recorded in two patients with extended lesions and in one with reduced oral hygiene and reduced adherence to the protocol schedule.

To conclude, the $\mathrm{O}_{2} / \mathrm{O}_{3}$ therapy with and without the debridement with piezoelectric surgery represents a promising approach to improve the treatment of BRONJ and therefore the life quality of the patients. Further studies should be conducted with the inclusion of a larger number of patients.

\section{Introduction}

Biphosphonate-related osteonecrosis of the jaw (BRONJ), recently re-named as medication-related osteonecrosis of the jaw (MRONJ), is the progressive death of bone cells caused by a reduced blood flow (osteonecrosis, ON) in the maxillofacial region of patients during and after biphosphonate therapy (long-term or high doses) ${ }^{[2] ~[3] ~[4] . ~ D e n t o a l v e o l a r ~}$ invasive surgery procedures such as implant insertions, periodontal surgery, surgical endodontics and tooth extractions are considered the main triggers for BRONJ. Anatomical alterations, dental prosthesis, inflammatory diseases of the oral 
cavity, smoking and other comorbidities (e.g. anemia, obesity) can also contribute to the development of this severe side effect of the bisphosphonate therapy [4] [5].

Among the investigated alternatives to the mainstream therapeutic approaches (antibiotic therapy and surgical removal) which have limited efficacy, oxygen/ozone $\left(\mathrm{O}_{2} / \mathrm{O}_{3}\right)$ therapy (a mixture of $\mathrm{O}_{2}$ and $\mathrm{O}_{3}$ is transferred to the body) has shown positive results ${ }^{[6]}[7]$. Indeed, $\mathrm{O}_{3}$ acts as a bactericidal agent and activates blood circulation by increasing the erythrocytes number and the hemoglobin concentration ${ }^{[6]}[8]$. At the Dentistry Department of the ASST Fatebenefratelli Sacco Hospital (Milan, Italy), an experimental protocol (approved by the Ethics Committee of Milano Area A) for the treatment of BRONJ with the $\mathrm{O}_{2} / \mathrm{O}_{3}$ therapy and the debridement with piezoelectric surgery has been followed since 2015. Preliminary data were recently published ${ }^{[1]}$. Here we report the updated case reports.

\section{Materials and methods}

Sixteen patients with BRONJ at stage 1 and 2 (classified according to the SICMF-SIPMO - Sistema di stadiazione clinica e radiologica) were included in the experimental protocol. The treatment was performed as described by Goker et al. $(2020)^{[1]}$. Before and following the debridement with piezoelectric surgery, the $\mathrm{O}_{2} / \mathrm{O}_{3}$ mixture was administered locally twice a week for 10 weeks with a 3-4 day interval between applications according to the SIOOT (Società Italiana di Ossigeno-ozono terapia) guidelines (Figure 1). Clinical (e.g. pain, edema, presence of inflammation and bacterial infection, photographs) and radiological (Orthopantomography (OPT) and Computerized axial tomography (TAC)) parameters were used to evaluate the lesions.

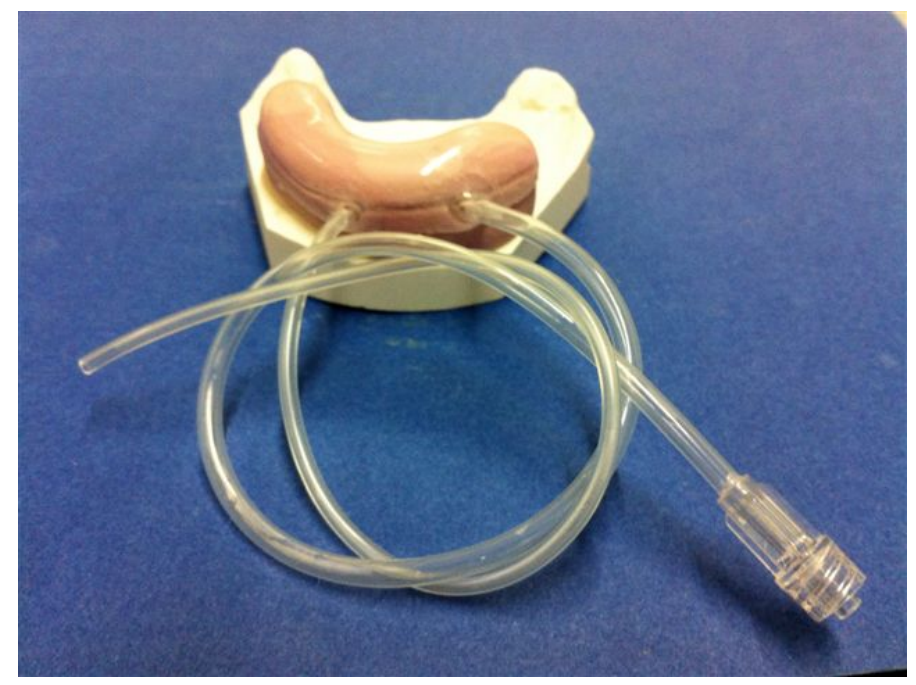

Figure 1. Application device.

\section{Results}

After the $\mathrm{O}_{2} / \mathrm{O}_{3}$ therapy, 12 of the 16 patients recovered from osteonecrosis and, in seven cases, the spontaneous expulsion of the bone fragment was observed (Table 1 and Figure 2). Thus, surgery procedures (debridement with piezoelectric surgery) were not necessary for six patients (two surgery procedures were planned for one of them). The 
lesions of one patient healed after two sessions: following relapses after the first $\mathrm{O}_{2} / \mathrm{O}_{3}$ session, the protocol was repeated with the recovery of the patient. In another case, the patient who had previously undergone the mainstream surgery procedures without any positive outcome was completely recovered after one $\mathrm{O}_{2} / \mathrm{O}_{3}$ session. Relapses were observed in two patients with extended lesions (the initial stage of the lesions was probably underestimated) and in one with reduced oral hygiene and reduced adherence to the protocol schedule (the patient would often postpone the appointments).

\begin{tabular}{|c|c|c|c|}
\hline \multicolumn{2}{|c|}{$\begin{array}{l}\text { Table 1. Overview of the } \\
\text { results obtained and of the } \\
\text { characteristics of the } 16 \\
\text { patients included in the } \\
\text { treatment. }\end{array}$} & $\begin{array}{c}\text { Healed } \\
\text { patients }\end{array}$ & $\begin{array}{c}\text { NOT } \\
\text { healed } \\
\text { patients }\end{array}$ \\
\hline \multicolumn{2}{|c|}{ TOTAL } & 12 & 4 \\
\hline \multicolumn{2}{|c|}{$\begin{array}{c}\text { of which spontaneous } \\
\text { expulsion }\end{array}$} & 7 & - \\
\hline \multicolumn{4}{|c|}{ Characteristics of patients } \\
\hline \multirow{2}{*}{ Age } & $40-60$ & 2 & 1 \\
\hline & $>60$ & 10 & 3 \\
\hline \multirow{2}{*}{ Gender } & $\mathrm{F}$ & 10 & 3 \\
\hline & $\mathrm{M}$ & 2 & 1 \\
\hline \multirow{2}{*}{ Pathology } & Neoplasia & 6 & 4 \\
\hline & Others & 6 & 0 \\
\hline \multirow{2}{*}{$\begin{array}{l}\text { MRONJ } \\
\text { area }\end{array}$} & Maxilla & 5 & 1 \\
\hline & Mandible & 7 & 3 \\
\hline
\end{tabular}

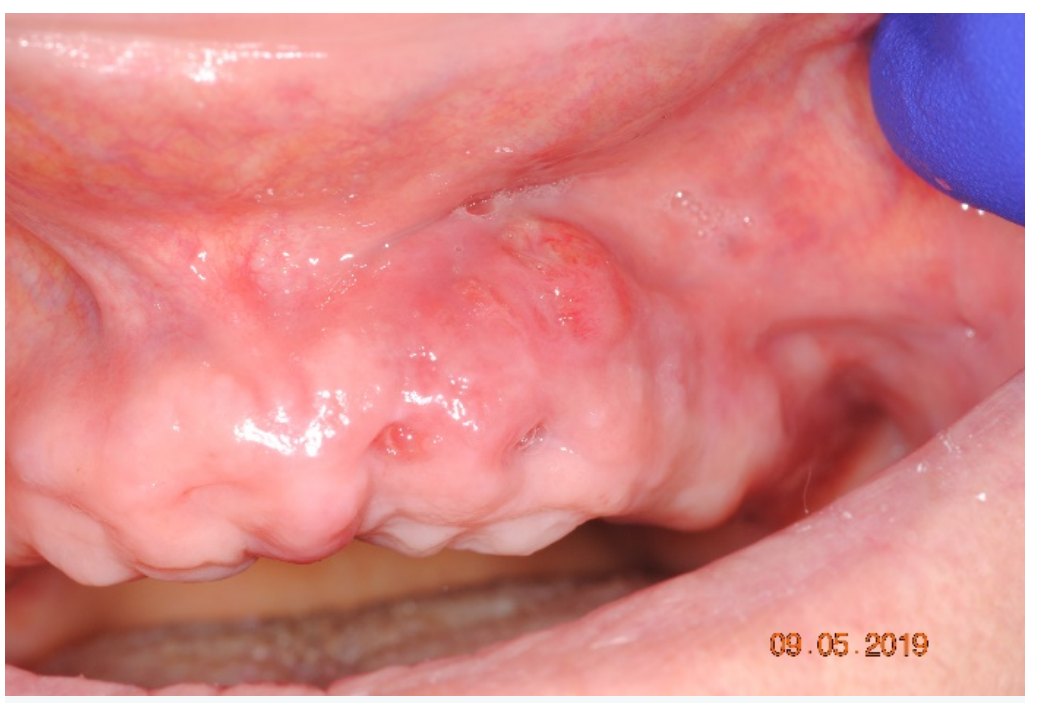

A 


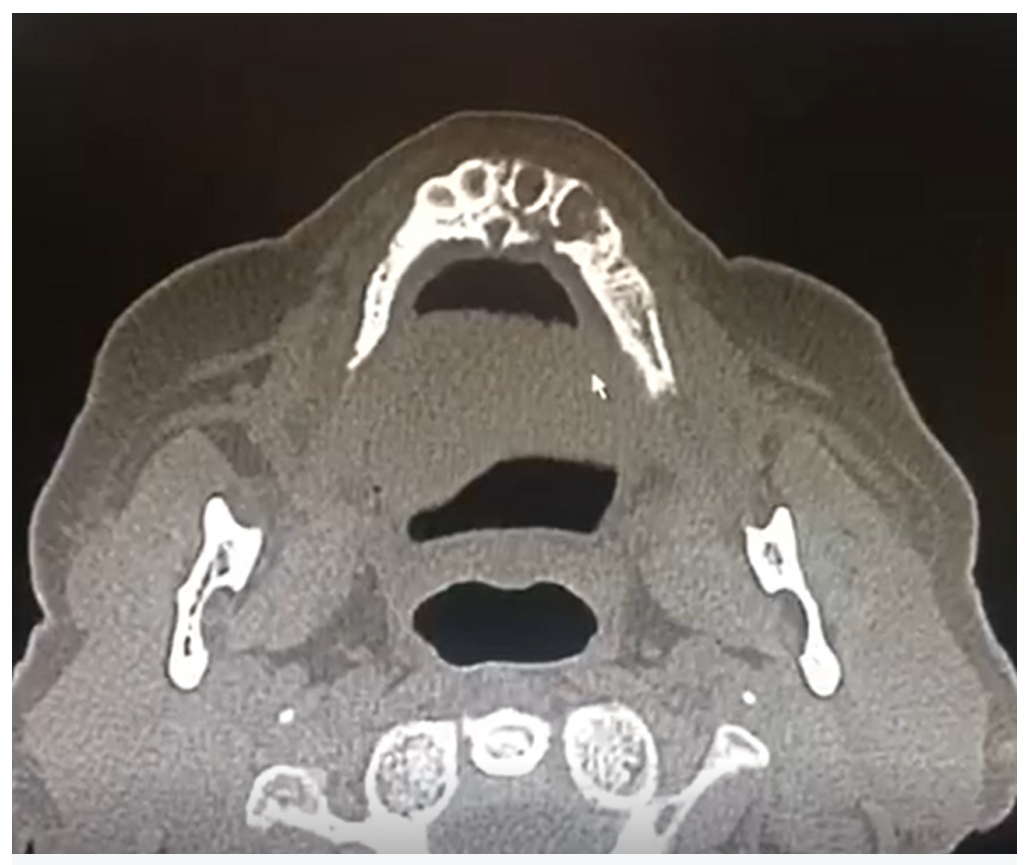

B

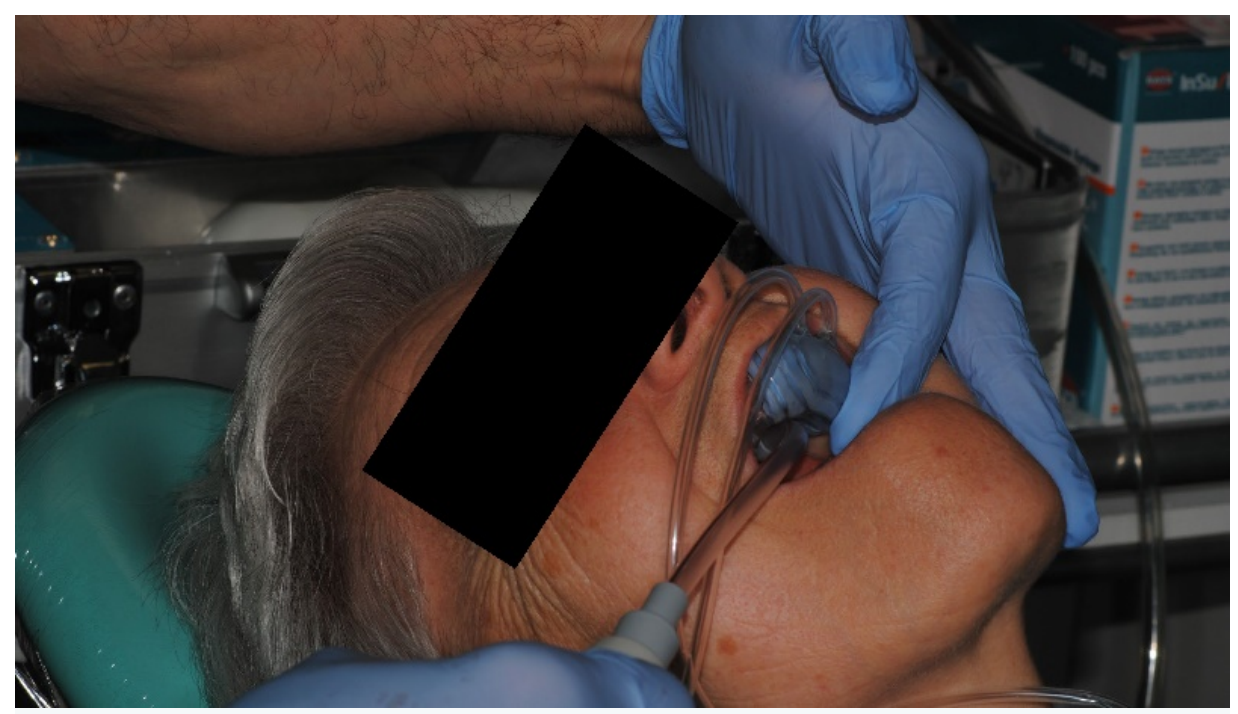

C 


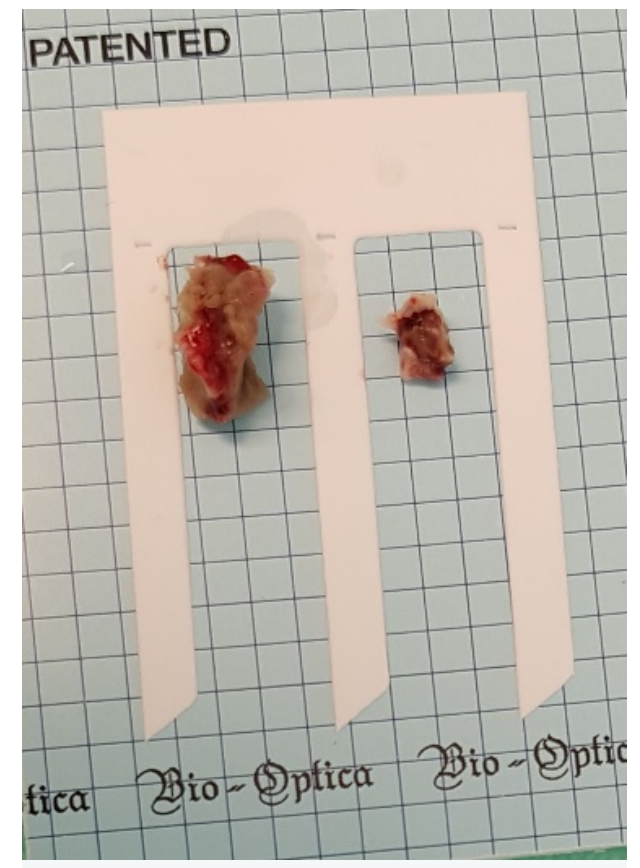

D

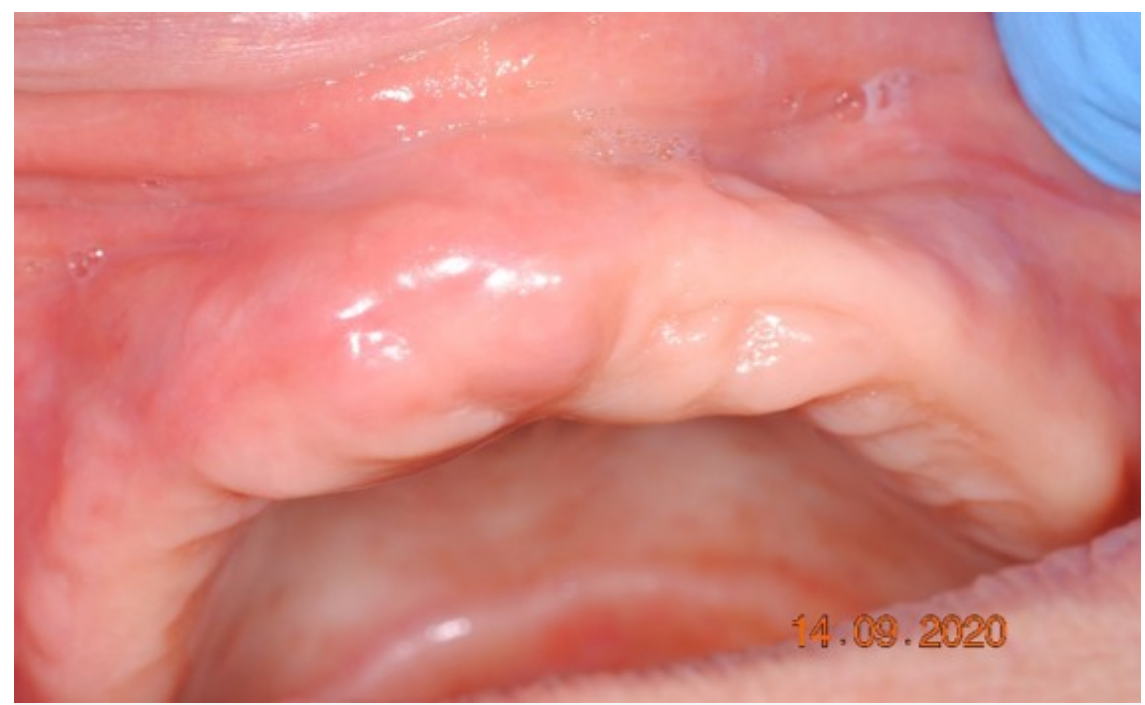

E

Figure 2. Case report. A, B) Initial status of BRONJ in one of the included patients (Maj 2019); C) Ozone/oxygen application; D) Spontaneous bone expulsion one month after the end of the $\mathrm{O}_{2} / \mathrm{O}_{3}$ therapy (September 2019); E) Complete healing of the lesion observed one year later (September 2020).

\section{Conclusions}

The $\mathrm{O}_{2} / \mathrm{O}_{3}$ therapy with and without the debridement with piezoelectric surgery represents a promising approach to improve the treatment of BRONJ and therefore the life quality of the patients. With the current experimental protocol, no side effects/adverse reactions to the $\mathrm{O}_{2} / \mathrm{O}_{3}$ mixture were recorded. The therapy was well tolerated in patients with compromised health conditions. Furthermore, the $\mathrm{O}_{2} / \mathrm{O}_{3}$ therapy has a low biological and economic impact thus representing a valuable therapeutic approach that could be considered/implemented as the mainstream therapy for the 
treatment of BRONJ. Further studies should be conducted with the inclusion of a larger number of patients.

\section{References}

1. a, b, c Funda Goker, Girolamo Donati, Francesco Grecchi, Antonella Sparaco, Mario Ghezzi, Vito Rania, Carlo A. Rossi, Massimo Del Fabbro. (2020). Treatment of BRONJ with ozoneloxygen therapy and debridement with piezoelectric surgery. European Review for Medical and Pharmacological Sciences, vol. 24 (17), 9094-9103.

2. ^Henry J. Mankin. (1992). Nontraumatic necrosis of bone (osteonecrosis). The New England Journal of Medicine, vol. 326, 1473-1479.

3. ^Robert E Marx. (2003). Pamidronate (Aredia) and zoledronate (Zometa) induced avascular necrosis of the jaws: a growing epidemic. Journal of Oral and Maxillofacial Surgery, vol. 61 (9), 1115-1117. doi:10.1016/s02782391(03)00720-1.

4. a, bSalvatore L. Ruggiero, Thomas B. Dodson, John Fantasia, Reginald Goodday, et al. (2014). American Association of Oral and Maxillofacial Surgeons Position Paper on Medication-Related Osteonecrosis of the Jaw-2014 Update. Journal of Oral and Maxillofacial Surgery, vol. 72 (10), 1938-1956. doi:10.1016/j.joms.2014.04.031.

5. 'Ourania Nicolatou-Galitis, Morten Schiødt, Rui Amaral Mendes, Carla Ripamonti, et al. (2019). Medication-related osteonecrosis of the jaw: definition and best practice for prevention, diagnosis, and treatment. Oral Surgery, Oral Medicine, Oral Pathology and Oral Radiology, vol. 127 (2), 117-135. doi:10.1016/j.00oo.2018.09.008.

6. a, b Alessandro Agrillo, Pierpaolo Sassano, Claudio Rinna, Paolo Priore, et al. (2007). Ozone Therapy in Extractive Surgery on Patients Treated With Bisphosphonates. doi:10.1097/scs.0b013e3181572609.

7. `Carla Ida Ripamonti, Enrico Cislaghi, Luigi Mariani, Massimo Maniezzo. (2011). Efficacy and safety of medical ozone (O3) delivered in oil suspension applications for the treatment of osteonecrosis of the jaw in patients with bone metastases treated with bisphosphonates: Preliminary results of a phase 1-Il study. Oral Oncology, vol. 47 (3), 185-190. doi:10.1016/j.oraloncology.2011.01.002.

8. 'Velio Alvaro Bocci. (2006). Scientific and Medical Aspects of Ozone Therapy. State of the Art. Archives of Medical Research, vol. 37 (4), 425-435. doi:10.1016/j.arcmed.2005.08.006. 